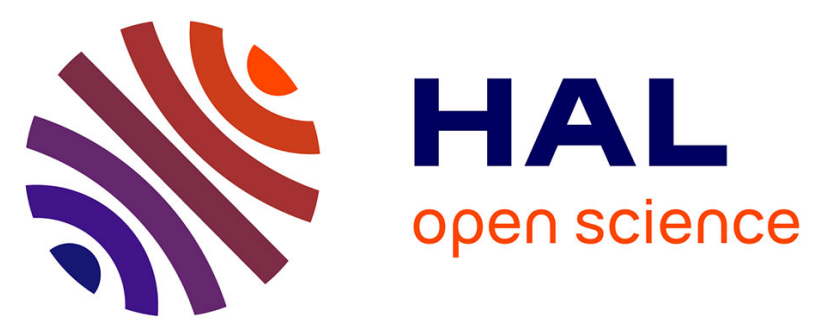

\title{
Concentrations de sables lourds sur les plages de la baie de Morlaix (Finistère) : Exemples de micro-placers d'origine proximale dans une profonde échancrure littorale en voie de déblaiement rapide par la mer
}

Louis Chauris

\section{To cite this version:}

Louis Chauris. Concentrations de sables lourds sur les plages de la baie de Morlaix (Finistère) : Exemples de micro-placers d'origine proximale dans une profonde échancrure littorale en voie de déblaiement rapide par la mer. Norois, 1984, 31 (123), pp.407-426. 10.3406/noroi.1984.7379 . insu01861009

\author{
HAL Id: insu-01861009 \\ https://hal-insu.archives-ouvertes.fr/insu-01861009
}

Submitted on 17 Feb 2021

HAL is a multi-disciplinary open access archive for the deposit and dissemination of scientific research documents, whether they are published or not. The documents may come from teaching and research institutions in France or abroad, or from public or private research centers.
L'archive ouverte pluridisciplinaire HAL, est destinée au dépôt et à la diffusion de documents scientifiques de niveau recherche, publiés ou non, émanant des établissements d'enseignement et de recherche français ou étrangers, des laboratoires publics ou privés.

\section{(ㅇ)(1) $\$$}

Distributed under a Creative Commons Attribution - NonCommercial - NoDerivatives $\mid 4.0$ 
Concentration de sables lourds sur les plages de la baie de Morlaix (Finistère): Exemples de micro-placers d'origine proximale dans une profonde échancrure littorale en voie de déblaiement rapide par la mer

Louis Chauris

\section{Citer ce document / Cite this document :}

Chauris Louis. Concentration de sables lourds sur les plages de la baie de Morlaix (Finistère): Exemples de micro-placers d'origine proximale dans une profonde échancrure littorale en voie de déblaiement rapide par la mer. In: Norois, $n^{\circ} 123$, JuilletSeptembre 1984. pp. 407-426;

doi : https://doi.org/10.3406/noroi.1984.7379

https://www.persee.fr/doc/noroi_0029-182x_1984_num_123_1_7379

Fichier pdf généré le 10/11/2019 


\begin{abstract}
SUMMARY

Investigations carried out upon heavy minerals (47 samples) concentrated along the beaches of the bay of Morlaix, a large coastal indentation in the Western Channel, have displayed seven different districts. As a general rule, the coastal placers present a proximal origin. The heavy sands come essentially, by marine erosion and sorting, from late continental sediments (arena and periglacial deposits, head). The outer contributions are small, except for the northwestern end of the bay and especially its northeastern part. The proximal origin of an important part of the sands could account for the future of the beaches, at present time altered by anthropic modifications.
\end{abstract}

\title{
Résumé
}

\section{RESUME}

L'étude des cortèges de minéraux lourds (47 prélèvements) concentrés sur les plages de la baie de Morlaix, profonde échancrure littorale de la Manche occidentale, permet de mettre en évidence sept districts différents. Dans l'ensemble, les placers littoraux présentent une origine proximale. Les sables lourds proviennent essentiellement de l'érosion et du tri par la mer, de dépôts continentaux récents (arènes, formations périglaciaires ou head). Les apports extérieurs à la baie restent limités, sauf à son extrémité nord-ouest et surtout dans sa partie nord-est. L'origine proximale d'une grande partie des sables contrôle le devenir des plages actuellement soumises à des modifications anthropiques. 
Norois, Poitiers, 1984, t. $31, \mathrm{n}^{\circ} 123$, p. 407-426.

\title{
Concentrations de sables lourds sur les plages de la baie de Morlaix (Finistère) :
}

\section{Exemples de micro-placers d'origine proximale dans une profonde échancrure littorale en voie de déblaiement rapide par la mer}

\author{
par Louis CHAURIS \\ Universite de Brest. \\ C.I.S. 410012 "Océanologie et Géodynamlque". \\ Département des Sciences de la Terre. Géologie. Faculté des Sciences 29283 Brest.
}

"O mer, ne reste-t-il que sable sur le sable pour écrire l'Histoire? "(Charles Le Quintrec)

\section{INTRODUCTION}

La provenance des sables lourds concentrés dans les placers littoraux pose souvent des problèmes délicats. Trois origines possibles s'offrent a priori : proximale, distale ou mixte. La genèse de ces occurrences est sous la dépendance de plusieurs facteurs successifs (Cronan, 1980) : (1) La source : roches-mères où les minéraux sont, soit disséminés dans des complexes éruptifs ou métamorphiques (ilménite, monazite, grenat...), soit déjà concentrés dans des filons (cassitérite...) (Tixeront, 1978) ; (2) L'altération de roches-mères sous des conditions climatiques favorables ; (3) Le transport par les eaux (fleuves côtiers, dérive littorale...), avec des possibilités de formations-relais ; (4) Le dépôt dans des pièges, liés à des conditions géomorphologiques particulières, sous l'influence des vagues, surtout en périodes de tempête (placers du type " haute énergie " (Moore, 1976), par triage sélectif dans la zone intertidale (Burns, 1979).

Dans le cadre des recherches systématiques entreprises depuis quelques années sur ces concentrations minérales qui ourlent l'Armorique comme un collier de gemmes déposé sur l'écrin des grèves (Chauris, $1982 \mathrm{a}, \mathrm{b}, \mathrm{c}$ ), on examine ici en détail une zone limitée, mais, à notre avis, fort instructive : la baie de Morlaix. Cette profonde échancrure de la Manche occidentale correspond à un environnement essentiellement "continental »: la mer

Mots-clés : Sédimentologie. Sables lourds. Plages. Baie de Morlaix. Massif armoricain. Key-words : Sedimentology. Heavy sands. Beaches. Bay of Morlaix. Armorican Massif. 
actuelle exhume, à vive allure, un relief fossilisé sous l'épais manteau des produits de la gélifraction et de la solifluxion würmiennes (Weichselien), saupoudré çà et là par une pellicule de sables dunaires.

Du point de vue de la morphogenèse littorale, qui est, en la matière, celui du géographe physicien, ce problème de provenance est capital, car il revient à se demander en quelle mesure et sur quelles distances les forces marines, et plus généralement les forces agissant dans le domaine littoral, peuvent transporter les sédiments dans un contexte morphostructural donné.

Le choix de cette zone a été guidé par de multiples raisons :

(1) Un arrière-pays très varié. La baie de Morlaix coïncide avec la limite de deux domaines géotectoniques différents : à l'ouest, le Pays de Léon, avec ses ensembles cristallophylliens (micaschistes, gneiss, amphibolites...) recoupés par de nombreux granitoïdes (diorites et divers granites) ; à l'est, le Petit-Trégor, avec ses ensembles basiques (épidiorites, gabbros...) traversés par quelques granites ; entre ces deux blocs, le fossé de Morlaix, avec ses formations schisteuses métamorphisées par les granites (Barrois, 1909 ; Sandréa, 1958 ; Chauris, 1972, 1978). Ces multiples roches-mères éventuelles permettent de bien poser le problème des zones-sources (filiation).

(2) Une morphologie littorale contrastée où les falaises (rocheuses ou limoneuses) alternent avec les côtes basses (sablonneuses ou vaseuses), sur les rives de deux profondes rias (la Penzé et la Rivière de Morlaix), de presqu'îles découpées et d'innombrables îlots.

(3) Des caractères hydrodynamiques heurtés : violents courants de marée, dus à un marnage qui peut dépasser 9 mètres en période de vives eaux; houles engendrées par des fetchs d'ampleur très inégale, très faibles (quelques kilomètres : secteur "sud ») ; relativement forts (de l'ordre de $200 \mathrm{~km}$ : secteur " nord »).

(4) Un cadre sédimentologique aux multiples facettes. Zones toujours immergées (Boillot, 1961, 1964), avec dépôts de sables terrigènes siliceux, phycogènes (maërl) et zoogènes d'origine variée. Zones intertidales (Bourcart, 1048, 1949 ; Francis-Bœuf, 1947 ; Auffret, 1968 ; Chauris-Norroy, 1973 ; Guilcher et al., 1957, 1982), caractérisées par une grande hétérogénéité de la couverture meuble, s'ajoutant à celle du substratum rocheux : cordons de galets, parfois de fière allure (Le Guerzit) pouvant ourler le haut de certaines plages ; l'émoussé, très fort dans les zones externes de la baie, diminue fortement vers les zones internes (passage aux cailloutis anguleux) ; plages de sables fins se poursuivant parfois au loin sur l'estran (Grève Blanche à Carantec); galets et sables sont souvent associés dans des flèches à pointe libre ; vastes étendues de vase (slikke) sur les berges des deux rias. La dérive littorale vers le sud est établie par la morphologie des flèches à pointe libre, et la migration des galets, des sables et des dépôts anthropiques (coquilles d'huîtres...).

Fic. 1. - Localisation des prélèvements de sables lourds (1 à 47) dans les micro-placers littoraux de la baie de Morlaix (Finistère).

En pointillí $=0$ de la carte marine. 


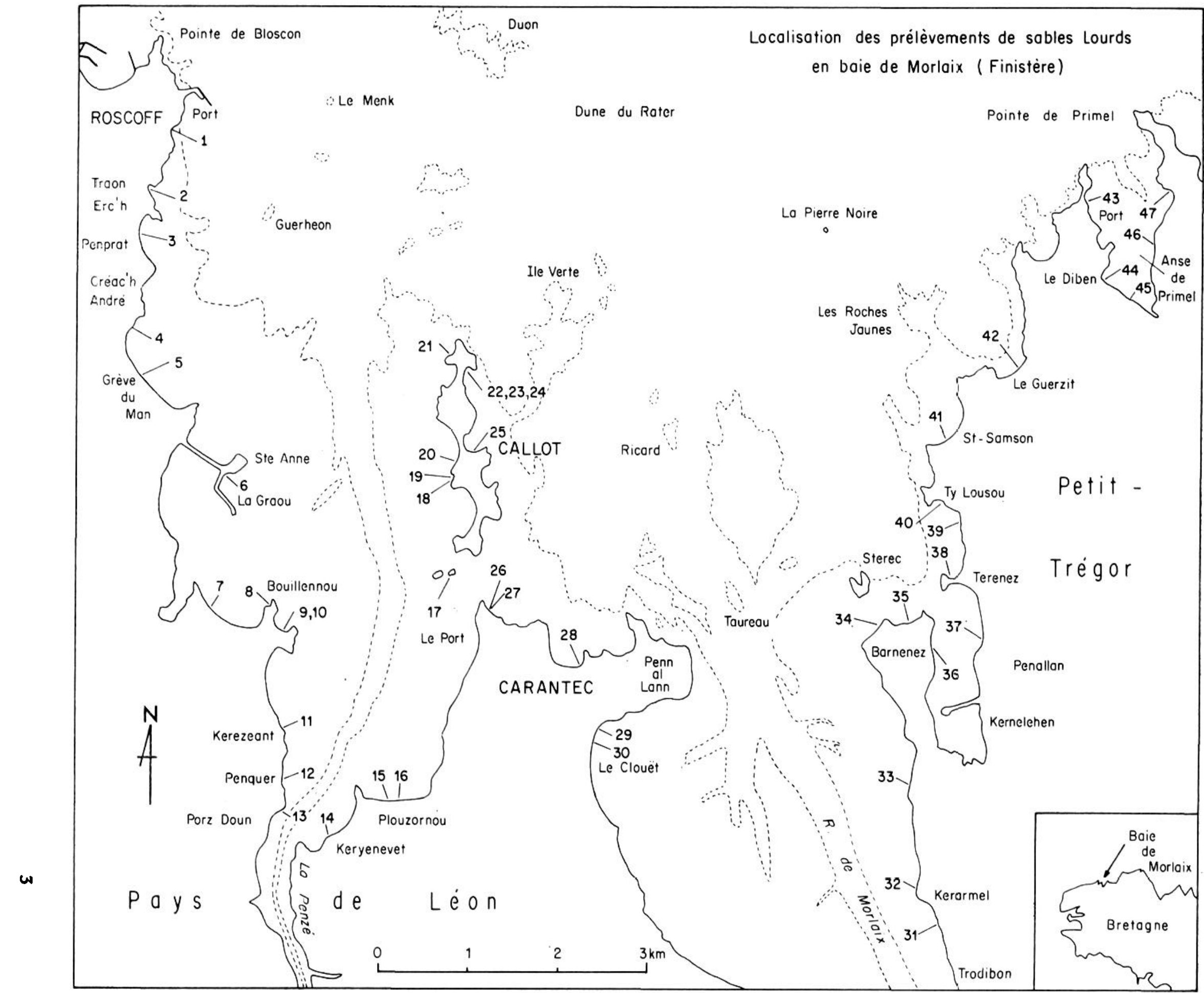


TAziEAU I. - Localisation des prélèvements de sables lourds. Délimitation des districts I à VII (voir texte).

$-=$ présent, $\operatorname{tr}=$ traces, $\mathrm{mir}=$ microtraces. Divers $(-$ ou $\mathrm{mtr}): \mathrm{Ba}=$ Barytine $; \mathrm{Au}=$ or natif $; \mathrm{As}=$ mispickel $; \mathrm{Cu}=$ Chalcopyrite ; $F=$ Fluorine.

\begin{tabular}{|c|c|c|c|c|c|c|c|c|c|c|c|c|c|c|c|c|c|c|c|c|c|c|c|c|c|}
\hline & I & & II . & Soint & - Pol - & Cle - Léo & & & & & III. & $\mathrm{Pe}$ & enze & & & & & & IV & - & Corant & & & & \\
\hline $\begin{array}{l}n^{0} \text { sur fig. } \\
\text { numéro } \\
\text { du } \\
\text { prélèvement }\end{array}$ & $\frac{1}{\stackrel{0}{\infty}}$ & 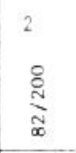 & ${ }_{\infty}^{3}$ & $\overbrace{\infty}^{4}$ & $\begin{array}{c}5 \\
2 \\
\frac{2}{5} \\
\infty\end{array}$ & 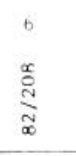 & $\sum_{\infty}^{\bar{\infty}}$ & $\underset{\infty}{\stackrel{8}{\alpha}}$ & $\begin{array}{l}3 \\
\frac{3}{2} \\
\end{array}$ & $\stackrel{m}{\infty}_{\infty}^{10}$ & $\underset{\substack{\infty \\
\infty}}{11}$ & $\begin{array}{l}: \\
\stackrel{2}{*} \\
\stackrel{2}{n}\end{array}$ & $\underset{\infty}{13}$ & $\frac{\bar{y}}{\bar{\alpha}}$ & 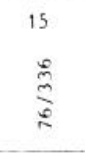 & $\frac{16}{\infty}$ & $\underset{\infty}{\stackrel{1}{+}}$ & $\underset{\infty}{18}$ & $\underset{\infty}{\stackrel{m}{\infty}}$ & $\frac{20}{\infty}$ & $\begin{array}{l}-1 \\
\underset{\infty}{\infty}\end{array}$ & $\frac{2}{2}$ & $\frac{23}{\stackrel{\alpha}{-}}$ & $\underset{\infty}{\stackrel{24}{\infty}}$ & $\stackrel{25}{\stackrel{25}{\infty}}$ \\
\hline $\begin{array}{l}\text { C ML } \\
\text { Cassitérite }\end{array}$ & 9,7 & $\begin{array}{l}13,8 \\
\text { mt r }\end{array}$ & 18,6 & 20,7 & $\begin{array}{l}24,4 \\
\text { mitr }\end{array}$ & 20.5 & 19,6 & 24,5 & $\begin{array}{l}64,6 \\
\text { metr }\end{array}$ & $\begin{array}{l}27,7 \\
\mathrm{mtr}\end{array}$ & $\begin{array}{l}84,6 \\
0,03\end{array}$ & 61,3 & $\begin{array}{l}66,9 \\
\mathrm{mtr}\end{array}$ & 63,5 & 57,1 & 40,7 & $\begin{array}{l}48,6 \\
0,13\end{array}$ & $\begin{array}{l}50,0 \\
0,01\end{array}$ & $\begin{array}{l}67,0 \\
\text { mtr }\end{array}$ & $\begin{array}{c}65,9 \\
\operatorname{tr}\end{array}$ & 26,3 & $\begin{array}{l}64,2 \\
\operatorname{mtr}\end{array}$ & $\begin{array}{l}40,5 \\
\mathrm{mtr}\end{array}$ & $\begin{array}{l}66,3 \\
\operatorname{tr}\end{array}$ & $\begin{array}{l}31,3 \\
\mathrm{mer}\end{array}$ \\
\hline Scheelite & & $\mathrm{tr}$ & metr & & $\mathrm{mtr}$ & metr & $\mathrm{mer}$ & mtr & mer & $\mathrm{mtr}$ & metr & & $\mathrm{mtr}$ & & & & & & & & mtr & $\mathrm{mtr}$ & & $\mathrm{mtr}$ & \\
\hline Tourma line & 25,2 & - & - & - & 1,9 & 2,4 & 1,5 & & - & - & 0,2 & - & - & - & - & - & - & - & - & & & & 2,5 & - & 1,1 \\
\hline Monazite & $m t r$ & $\mathrm{tr}$ & & ntr & 0,9 & mtr & $\mathrm{tr}$ & & 3,1 & 1.0 & tr & metr & $\mathrm{mtr}$ & & & nttr & 3.5 & 2,0 & 2,9 & 4,9 & tr & 1,8 & 3,3 & 2,8 & 2,2 \\
\hline Rutile & - & - & - & - & 0,9 & - & - & - & - & - & 0,2 & - & - & - & - & - & - & - & - & - & - & - & - & - & - \\
\hline Anatase & - & - & & - & - & - & - & - & - & - & - & - & - & - & - & - & - & - & - & - & - & - & - & - & - \\
\hline Zircon & - & - & - & - & 1,0 & - & 2,0 & - & - & 1,0 & 0,6 & - & - & - & 0,8 & 1,1 & 1,6 & 1,7 & 4,4 & 4,9 & 2,0 & 0,5 & 9,9 & 5,6 & 5,5 \\
\hline Nagnetite & 10,1 & - & - & 1,7 & 1,0 & - & - & - & 2,0 & 2,5 & 0,6 & - & 0,5 & 10,1 & 0,8 & - & 10,3 & 2,7 & 2,9 & - & 9,9 & 2,6 & 4,1 & 2,8 & 1,6 \\
\hline Ilménite & - & 14,8 & 3,5 & - & 10,3 & 9,5 & 19,9 & 19,0 & 84,0 & 86,6 & 80,7 & 42,4 & 40,7 & 81,8 & 60,6 & 70,1 & 76,9 & 84,8 & 73,7 & 73,5 & 76,8 & 87,3 & 57,1 & 70,7 & $78, \dot{2}$ \\
\hline Grenat & 16,8 & 9,1 & 9,3 & 3,5 & 67,7 & 41,8 & 9,9 & 1,6 & 2,0 & 4,2 & 8,8 & 8,2 & 4,0 & - & 1,6 & 7.9 & 4,3 & 8,2 & 8,8 & 4,9 & 3,3 & 4,2 & 3,3 & 5,6 & 7,1 \\
\hline Apatite & - & - & - & 1,7 & & - & - & - & - & - & - & - & - & - & - & - & & & - & - & 1,2 & - & 1,6 & - & - \\
\hline Staurotide & 8.4 & 3,4 & 9.3 & 6,1 & 14,8 & 28,7 & 1,5 & - & - & 1,3 & 1,4 & - & - & - & - & - & 0,8 & & - & - & - & - & - & - & 1,1 \\
\hline Disthène & - & - & - & - & - & - & - & - & - & - & - & - & - & - & - & - & - & - & - & - & - & - & - & - & - \\
\hline Sillimanite & - & - & - & - & - & - & - & - & - & & & & & & & & & - & & - & & - & - & & - \\
\hline Andalousite & - & - & - & - & - & - & - & - & - & - & - & & - & & & - & - & - & - & - & - & - & & - & - \\
\hline Amphibole & 33,6 & 65,9 & 70,9 & 83,5 & - & 14,3 & 62,7 & 77,8 & 5,1 & 3,0 & 5,8 & 41,3 & 53,0 & 6,1 & 29,5 & 10,2 & 1,6 & - & - & - & 3,3 & 1.0 & 16,5 & 2,8 & 1,6 \\
\hline Epidote & - & - & 5,8 & - & & - & - & - & - & - & 1,5 & - & 0,5 & - & 5,3 & 7,9 & - & - & - & - & - & - & - & - & - \\
\hline Sphène & - & - & - & 1,7 & & & - & - & - & & - & & - & & - & - & & & - & - & 0,6 & - & - & - & \\
\hline Pyrite & - & & & & - & - & - & & & & - & & - & & & & & - & & - & - & & & & \\
\hline Corindon & - & & - & & - & - & - & & - & - & - & & & & & & - & & - & - & & - & & - & - \\
\hline Biotite & - & - & & & & & & & & & & & & & & & & & - & - & 2,0 & & - & - & - \\
\hline Divers (mt $r$ ) & & & & & & & & & & & & & & & & & $\mathrm{Ba}$ & & Au & & & & & $\mathrm{Au}$ & \\
\hline
\end{tabular}




\begin{tabular}{|c|c|c|c|c|c|c|c|c|c|c|c|c|c|c|c|c|c|c|c|c|c|c|}
\hline \multirow{3}{*}{$\begin{array}{l}\mathrm{n}^{\circ} \text { sur fig. } \\
\text { numéro } \\
\text { du } \\
\text { prélèvement }\end{array}$} & \multicolumn{5}{|c|}{ IV - Corantec } & \multicolumn{3}{|r|}{$\bar{Y}$} & \multicolumn{2}{|l|}{ - } & \multicolumn{4}{|c|}{ Plouézoc'h } & \multicolumn{4}{|c|}{ II Le Diben } & \multicolumn{4}{|c|}{ VIII Primel } \\
\hline & .26 & 27 & 28 & 29 & 30 & 31 & 32 & 33 & 34 & 35 & 36 & 37 & 38 & 39 & 40 & 41 & 42 & 43 & 44 & 45 & 46 & 47 \\
\hline & $\frac{\bar{\infty}}{\infty}$ & $\frac{\hat{\stackrel{0}{ }}}{\bar{\infty}}$ & $\underset{\infty}{\stackrel{\infty}{\sim}}$ & $\stackrel{\infty}{\stackrel{\infty}{\infty}}$ & 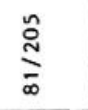 & $\underset{\infty}{\stackrel{n}{\curvearrowright}}$ & $\underset{\infty}{\stackrel{\sim}{\sim}}$ & 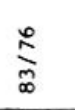 & $\underset{\infty}{\stackrel{\tilde{N}}{\alpha}}$ & $\underset{\infty}{\stackrel{\infty}{ٍ ̆ ~}}$ & $\stackrel{\hat{\infty}}{\hat{\infty}}$ & $\stackrel{\infty}{\stackrel{\infty}{\infty}}$ & $\underset{\infty}{\stackrel{\sim}{\sim}}$ & $\stackrel{\infty}{\stackrel{\infty}{\curvearrowright}}$ & $\underset{\infty}{\stackrel{\infty}{\check{~}}}$ & 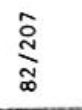 & $\stackrel{\infty}{\infty}$ & $\sum_{\infty}^{\infty}$ & $\frac{5}{\infty}$ & $\underset{\infty}{\stackrel{\infty}{\infty}}$ & $\underset{\infty}{\stackrel{\infty}{-\infty}}$ & $\stackrel{n}{\infty}$ \\
\hline $2 \mathrm{ML}$ & 32,8 & 50,0 & 22,1 & 65,5 & 32,0 & 61,0 & 41,3 & 59,3 & 37,0 & 31,8 & 27,4 & 20,0 & 17,8 & 19,8 & 21,9 & 16,2 & 8,4 & 9,5 & 45,8 & 33,8 & 42,9 & 65,2 \\
\hline Cassitérite & 0,11 & 0,85 & $\mathrm{mtr}$ & $\mathrm{mtr}$ & mtr & & $\mathrm{mtr}$ & mtr & & & & & & 0,25 & metr & & mer & & mtr & mtr & & \\
\hline Seheelite & $\mathrm{mtr}$ & $\mathrm{mtr}$ & $\mathrm{mtr}$ & & mer & & & & & & & & & & & & mtr & & & & & \\
\hline Tourmaline & - & 0,6 & - & - & 0,9 & - & - & 0,8 & - & - & - & - & 0,9 & 1,2 & 7,4 & 1,3 & 4,3 & 5,9 & - & - & - & - \\
\hline Monazite & 4,3 & 5,1 & 5,7 & 2,8 & 1,3 & $\mathrm{mtr}$ & $\mathrm{mtr}$ & $\mathrm{mtr}$ & & & & $\mathrm{mtr}$ & & $\operatorname{tr}$ & 14,9 & 1,3 & $\operatorname{tr}$ & tr & tr & $\mathrm{mer}$ & , & $\mathrm{mtr}$ \\
\hline Rutile & - & 0,6 & - & - & 0,9 & - & - & - & - & - & - & - & - & - & - & - & - & - & & - & - & - \\
\hline Anatase & - & & - & - & - & - & - & - & - & - & - & - & - & - & - & - & & & & - & - & - \\
\hline Zircon & 2,2 & 0,6 & - & - & 1,3 & - & - & - & - & - & - & - & 2,9 & " & 4,5 & 2,6 & 3,2 & 4,4 & 3,1 & 2,9 & - & 1,5 \\
\hline Magnétite & 4,3 & 6,8 & - & - & 0,9 & 1,6 & - & 0,8 & - & - & - & 4,7 & 3,8 & - & - & - & - & 11,7 & 0,8 & 1,1 & 3,1 & 3,7 \\
\hline Ilménite & 69,5 & 79,6 & 84,8 & 81,9 & 77,2 & 32,2 & 40,0 & 36,9 & 11,5 & 12,2 & 21,4 & 37,5 & 38,4 & 46,8 & 58,2 & 85,3 & 52,3 & 52,9 & 92,8 & 85,2 & 65,6 & 82,1 \\
\hline Grenat & 6,5 & 4,2 & 3,4 & 8,5 & 14,3 & 1,6 & - & 1,6 & - & - & - & - & 3,8 & 8,8 & 4,4 & 1,3 & 6,5 & 5,9 & - & & - & - \\
\hline Apa:ite & - & - & - & - & & & & & - & & & - & & & - & - & - & & & & & \\
\hline Staurotide & - & - & - & - & - & - & - & - & - & - & - & - & & - & & & 4,3 & & & & & \\
\hline Disthène & - & - & - & - & & - & & - & - & - & & - & - & & - & - & - & - & - & - & - & \\
\hline Sillimanite & - & - & - & - & & & - & - & - & & & & - & - & - & - & & - & - & - & & - \\
\hline Andalousite & - & & - & - & 1,3 & 1,3 & - & 0,8 & - & - & 7,1 & - & - & - & - & 1,7 & 3,2 & - & - & - & & \\
\hline Amphibole & 8,7 & 0,6 & - & - & 0,9 & 32,2 & 26,6 & 34,8 & 82,7 & 77,4 & 53,5 & 37,5 & 24,0 & 20,2 & 5,9 & 3,4 & 13,0 & 11,7 & 1,4 & 5,7 & 24,7 & 6,1 \\
\hline Epidote & & - & - & - & - & 29,0 & 26,6 & 22,8 & 4,6 & 9,1 & 14,3 & 15,6 & 24,0 & 20,2 & 3,0 & 1,7 & 6,5 & 7,3 & 1,4 & 4,5 & 6,2 & 6,0 \\
\hline Sphène & - & - & & - & & & & & & & & - & & & & & - & & & & & \\
\hline Pyrite & & & & & & & - & & - & & & & & - & & - & - & & & & & \\
\hline Corindon & - & & - & - & & & - & - & - & & - & & - & - & & - & - & & - & - & - & \\
\hline Biotite & & & & & & & & & & & & & & - & & & & & & & & \\
\hline Divers ( $\mathrm{mtr}$ ) & & & & & & & & & & & & & & & & & As & $\mathrm{Cu}, \mathrm{F}$ & & & & \\
\hline
\end{tabular}


(5)L'absence d'études antérieures sur les minéraux lourds, mises à part quelques observations très ponctuelles (Duplaix, 1946 ; Deunff, 1951). Les travaux classiques sur les minéraux lourds de la Manche sont à une toute autre échclle (Balk, 1936 ; Berthois et Furnestin, 1937 ; Duplaix et Boillot, 1968) ou se rapportent à des districts différents (Lafon, 1953 ; Billy, 1964 ; Giresse et Larsonneur, 1970 ; etc...). Seul le travail de Francis-Bœuf, Bourcart et Duplaix (1949) sur l'Aber de Roscoff, situé juste à l'ouest de notre domaine d'étude, a été effectué à une échelle très détaillée, mais il est regrettable que les minéraux opaques (ilménite, magnétite...) n'aient pas été déterminés. Une première publication relative au nord-ouest de la Bretagne (Chauris, 1982b) montrait la nécessité d'analyser un plus grand nombre d'échantillons pour mieux cerner les limites des districts déjà entrevus et confirmer les premières hypothèses.

Dès le début de nos recherches en baie de Morlaix, l'utilisation systématique des minéraux lourds (grand nombre de micro-placers disséminés sur les parties hautes des estrans) suggérait de poser un certain nombre de questions (1). Les associations minéralogiques révèleront-elles une certaine uniformité à l'échelle de la baie ou, au contraire, une succession de petites cellules de composition différente, d'origine locale, reflétant (et sous cutelle forme) la nature de l'arrière-pays (et éventuellement de l'avantpays) attaqué par l'érosion ? (2) Sera-t-il possible de préciser la part d'éventuels apports du large vis-à-vis des apports côtiers (influence respective offshore - onshore dans la balance sédimentologique) ? (3). La détermination de l'origine des sables lourds concentrés sur les plages permettra-t-elle de pressentir le devenir de ces plages, à la suite des modifications anthropiques des rivages actuellement en cours dans plusieurs secteurs?

\section{I. - MISE EN EVIDENCE DE SEPT DISTRICTS.}

La côte a été prospectée pas à pas depuis la pointe de Bloscon, à l'ouest, jusqu'à la pointe de Primel à l'est. En vue de mieux situer les placers de la baie de Morlaix, des recherches préliminaires ont été entreprises également plus à l'ouest (île de Batz, Roscoff, Santec) et plus à l'est (en direction de Saint-Jean-du-Doigt et de Guimaëc). Vers le sud, les accumulations sableuses disparaissent : dans la Penzé, aux environs de Porz Doun et du Lingos ; dans la Rivière de Morlaix, au droit de Locquénolé et du Dourduff. Certaines parties du littoral ont fait l'objet d'observations échelonnées sur plusieurs années et à différentes saisons. Les prélèvements (de l'ordre de $500 \mathrm{~g}$ à $1 \mathrm{~kg}$ par échantillon) ont été effectués à la pelle, par raclage des placages minéralisés, toujours localisés en haut de plage (zones de concentration naturelle en sables noirs, éventuellement rouges ou verts). Au total, 47 échantillons ont été analysés selon la méthode décrite dans les ouvrages de Guigues et Devismes (1969) et de Devismes (1978). Le pourcentage des minéraux lourds $(\mathrm{d}>2,9)$ par rapport au sable brut, est donné en poids ; le pourcentage des différents minéraux lourds (M.L.) est également donné en poids. Le cortège des M.L. est généralement varié : dans chaque échantillonnage, 11 à 20 minéraux ont été déterminés. En règle générale, le spectre minéralogique est caractrisé par quelques espèces qui lui confèrent un cachet particulier. Sept districts différents ont pu être ainsi distingués (fig. 1 ; tableau I, 1 à 47). 


\section{1. - DISTRICT DE ROSCOFF (1, 1).}

Cóte rocheuse (granite porphyroïde de Roscoff), avec grèves peu nombreuses, ourlées de galets bien roulés. Les dépôts de sables lourds sont de très faible extension et réduits à de minces placages. L'unique prélèvement (1) (Pen ar Creac'h) se distingue immédiatement de tous les autres échantillons de la baie de Morlaix par sa forte teneur en tourmaline ( $25 \%$, ), alors que partout ailleurs, les teneurs sont le plus souvent inférieures à $1 \%$ (sauf dans le district VI, où elles peuvent atteindre $7 \%$ ) ; le pourcentage en M.L. est relativement très bas $(9,7 \%)$. Par contre, Je spectre minéralogique et le pourcentage en M.L. de l'occurrence de Pen ar Créac'h sont assez comparables à ceux observés immédiatement plus à l'ouest, dans la région de Roscoff (Chauris, en préparation), où les teneurs moyennes de 8 échantillons en tourmaline, grenat et amphibole sont respectivement de $11,8 \%, 13,8 \%$ et $41,9 \%$. Ce prélèvement septentrional traduit ainsi la migration, par le nord-ouest, dans la baie de Morlaix, de sables d'origine plus occidentale, en provenance des grandes accumulations sableuses de l'ouest de Roscoff. Une telle origine, relativement distale, s'avère assez exceptionnelle dans la baie (cf. également le district VI).

\section{2. - DISTRICT DE SAINT-POL-DEৎLEON (II, 2 à 8).}

Entre la grève de Traon Erc'h et l'ouest de la pointe de Bouillennou. Le granite de Roscoff, avec ses différenciations dioritiques (Créac'h André) fait place vers le sud-est à un granite grossier à deux micas (Sainte-Anne). Les granites sont intrusifs dans un socle cristallophyllien complexe (quarı̇ites, micaschistes à grenat et staurotide, gneiss, amphibolites...). Côte parfois rocheuse, ou formée de falaises limoneuses ou encore ourlée de petites dunes, prolongées par un large estran sableux ; grandes plages (1 $\mathrm{km}$ à la Grève du Man), orientées préférentiellement au nord-est.

Les placages minéralisés sont assez. fréquents, mais le plus souvent, de faible inportance, sauf à la Grève du Man où les dépôts sont visibles sur plusieurs centaines de mètres. Les pourcentages en M.L. restent toujours relativement faibles $(13,8$ à $24,5 \%$; teneur moyenne $=20,3 \%)$. La teneur en tourmaline est basse ( $\max .2,4 \%$ ), soit au moins 10 à 20 fois inférieure à celle du district de Roscoff. L'influence des apports occidentaux s'atténue ici très rapidement puisqu'elle n'est plus décelable à Traon Erc'h à moins de 2 kilomètres au sud de la pointe de Bloscon. Les teneurs en ilménite sont remıarquablement basses (toujours inférieures à $20 \%$; teneur moyenne $=11 \%$ ) et, ainsi, de loin, (avec celles du district de Roscoff), les plus faibles de toutes celles de la baie de Morlaix. Le contraste frappant avec le district III (Penzé) montre qu'il n'y a pas d'apport du Sud vers le Nord. Les teneurs en grenat sont les plus élevées de la baie; les M.L. de la Grève du Man sont, pour les deux tiers, constitués de grenat : c'est ici la seule zone où les sables soient nettement rougeâtres. A l'évidence, les grenats proviennent des micaschistes grenatifères dont affleure ici l'extrême prolongement oriental de la formation du Conquet (Bas-Léon). C'est à la même roche-mère qu'il faut attribuer l'origine des staurotides (jusqu'à plus du quart des M.L. à Sainte-Anne). La très forte teneur en amphibole du prélèvement 4 (Creac'h André) coïncide manifestement avec la présence de la diorite voisine. Cette origine proximale est appuyée par 
la morphoscopie très anguleuse du minéral et, aussi, par la présence de sphène (de même origine dioritique) en quantité significative. L'origine locale des autres minéraux précités est également appuyée par leur morphoscopie le plus généralement anguleuse. La mise en évidence de scheelite $\left(\mathrm{WO}_{4} \mathrm{Ca}\right)$ dans six échantillons est à rapporter à des bancs de pyroxénites minéralisées, récemment découverts (Chauris, inédit). A l'extrémité méridionale du district II, les prélèvements 7 et 8 révèlent une chute des teneurs en grenat et surtout en staurotide qui annonce le district III.

\section{3. - DISTRICT DE LA PENZE (III, 9 à 16).}

De la pointe de Bouillennou à la Grande Grève de Plouzornou, de part et d'autre de l'estuaire de la Penzé. Le substratum est principalement constitué par des formations cristallophylliennes (amphibolites gneissiques dominantes, gneiss, micaschistes, quartzites), recoupées par l'extrême bordure sud-ouest de granite rose de Carantec (Pointe Saint-Jean). Côte souvent basse, en voie de recul très rapide dans les zones limoneuses attaquées en falaises ébouleuses, prolongée par un estran généralement vaseux. En plusieurs points, le limon affleure directement sur les grèves, sans aucun recouvrement sableux.

Le pourcentage en M.L. augmente considérablement par rapport au district II (teneur moyenne $=58,3 \%$ ). C'est ici que se rencontrent à la fois les concentrations les plus élevées en M.L. $(84,6 \%$ à Kerezéant, prélèvement 11) et les plus importantes accumulations de sables noirs de toute la baie de Morlaix : entre Kerezéant et Porz Doun, les dépôts massifs qui atteignent plusieurs centimètres de puissance sur le haut de l'estran, s'échelonnent d'une manière presque continue sur plus de 800 mètres. L'ilménite est, de très loin, le minéral prédominant (parfois $>85 \%$ ). Le contraste avec le district II est encore renforcé par la nette diminution de la teneur moyenne en grenat $(4,6 \%)$ et surtout en staurotide $(<1 \%)$. L'amphibole est toujours présente mais avec des pourcentages extrêmement variables ( 3 à $53 \%$ !). La provenance de l'amphibole et de l'ilménite à partir des amphibolites gneissiques est attestée, à l'évidence, par la morphoscopie extrêmement anguleuse de ces deux minéraux. La très faible tencur en tourmaline confirme l'origine locale des sables.

\section{4. - DISTRICT DE CARANTEC (IV, 17 à 30).}

Forme toute la presqu'île de Carantec et l'île Callot qui la prolonge vers le nord. Le granite rose à biotite recoupe à l'emporte-pièce un socle cristallophyllien très varié (gneiss, amphibolites, micaschistes, quartzites, schistes) qui constitue des lambeaux disloqués de toute dimension. La côte est généralement basse, sauf en quelques points (Penn al Lann...). Promontoires rocheux alternent avec grèves sablonneuses au fond d'anses creusées dans des limons souvent tapissés de petites dunes. Au sud, les grèves se prolongent par des vasières; au nord, par de vastes étendues de sable. Au sud du port de Carantec, le développement des installations ostréicoles (avec accumulations de coquilles d'huîtres) rend, à présent, les observations minéralogiques impossibles. 
Les dépôts de sables noirs sont nombreux, mais, le plus souvent, de petite dinension. A Penn ar Warem (Callot), une quinzaine de couches de un millimètre à quelques millimètres a été observée sur $\sim 30$ centimètres de profondeur, jusqu'au bed-rock (limon). Généralement, seule une partie de la plage est minéralisée : ainsi, au Clouët, la partie septentrionale. Les concentrations en M.L. sont assez élevées (teneur moyenne $=47,3 \%$ ), mais moindres toutefois que dans le district précédent. Le district IV est caractérisé minéralogiquement par des teneurs relativement fortes en monazite (jusqu'à $5,7 \% ;$ moyenne $=3 \%$ ), en zircon (jusqu'à $9,9 \%$; moyenne $2,9 \%$ ) et en magnétite (jusqu'à 10,3\%; moyenne $=3,5 \%$ ). Les teneurs en ilménite sont très élevées (jusqu'à 87,3 ; moyenne $=: 76,5 \%$ ). La chute des pourcentages en amphibole est très nette : la teneur moyenne $(2,6 \%)$ est, de loin, la plus faible notée dans toute la baie de Morlaix. Les teneurs en tourmaline et en staurotide sont très faibles; les teneurs en grenat généralement peu élevées.

L'examen morphoscopique de divers minéraux du district IV révèle des données à première vue contradictoires. Si, dans l'ensemble, le caractère anguleux domine, l'émoussé est parfois indubitable. Le cas de la magnétite est exemplaire : ce minéral apparaît, dans les mêmes prélèvements, à la fois cn grains anguleux (octaèdres), mais aussi en grains plus ou moins roulés. La même remarque s'applique à l'ilménite... Ces observations peuvent s'interpréter de la manière suivante. Par suite de sa situation, le district de Carantec est relativement exposé aux houles du large. Une certaine usure s'est produite pour les minéraux dispersés sur les estrans depuis un certain laps de temps, tandis que les minéraux libérés récemment de leur roche-mère (ou des formations-relais) n'ont pas eu encore le temps d'acquérir cette usure. Un émoussé prononcé ne peut, en luimême, prouver une origine distale, mais il peut suggérer un brassage prolongé.

L'origine essentiellement locale des sables lourds du district IV semble attestée par l'étude récente de certains minéraux. La cassitérite $\left(\mathrm{SnO}_{2}\right)$ fournit un premier exemple. Ce minéral est relativement fréquent dans les placers de la baie de Morlaix : 26 prélèvements sur 47 l'ont mis en évidence, mais son importance relative est variable selon les districts. En fait, la cassitérite est présente, d'une manière préférentielle, dans le district IV où elle a été notée dans 13 prélèvements sur 14 , avec des teneurs parfois relativement fortes (jusqu'à $0,85 \%$ ) dans certains placages. Ce minéral a été découvert en place dans des filons de quartz intragranitiques en plusieurs points, dans les falaises de Carantec (Chauris, 1975). Son origine est ainsi, au moins en partie, proximale. Cette manière de voir est confirmée par une autre observation : le seul autre point de la baie de Morlaix oì la cassitérite a été décelée avec des teneurs significatives $(0,25 \%)$ est situé dans le district $\mathrm{V}$ (Plouezoc'h) où affleurent, à proximité du prélèvement, et en bordure de mer, des greisens stannifères. Un deuxième exemple est donné par le grenat : la seule teneur élevée du district IV a été notée au Clouët (échantillon 30 ) où affleurent des schistes localement très grenatifères. C'est dans le même prélèvement que l'andalousite - observée dans les mêmes schistes du Clouët - a été décelée avec une teneur significative $(1,3 \%)$. Un dernier exemple peut être représenté par la monazite qui paraît bien être liée au granite de Caran- 
tec : les teneurs notables apparaissent dès les premiers affleurements de ce granite (prélèvement 9 dans le district III) et décroissent avec l'éloignement du granite (prélèvement 30 , Le Clouët).

\section{5. - DISTRICT DE PLOUEZOC'H (V, 31 à 39).}

De Trodibon au nord de Terenez. Essentiellement constitué de « roches vertes " (gabbros et épidiorites), recoupées au nord pār le leucogranite de Strrec. Falaises fréquentes, souvent entaillées dans le head et les limons et alors vivement attaquées par la mer. Larges estrans, vaseux au sud, sableux au nord. Ce district se distingue nettement de l'ensemble IV par la faible importance des placers, la nette diminution des teneurs en M.L. et surtout les puurcentages différents dans les associations. Monazite à l'état de microtraces, voire inexistante. Très nette diminution des teneurs en liménite $(30,7 \%)$, en magnétite et en grenat. Inversement, teneurs élevées en amphibole $(43,2 \%)$. Les fortes teneurs en épidote (jusqu’à $29 \%$; teneur moyenne $=18,4 \%)$ singularisent ce district parmi tous les autres cnsembles de la baie de Morlaix. Avec l'amphibole, ce minéral confère parfois aux sables une teinte verdâtre. Ces deux minéraux verts proviennent manifestement des " roches vertes " voisines, comme le confirme l'examen morphoscopique. L'origine de l'andalousite est à rechercher dans les schistes métamorphiques dont des panneaux enclavés au sein du granite ont été découverts dans les îlots de la baie (Chauris, inédit). La forte teneur du prélèvement $36(7,1 \%)$ pourrait traduire l'influence de la dérive littorale vers le sud. Comme on l'a vu plus haut, la cassitérite du prélèvement 39 provient des greisens stannifères situés à proximité (Terenez).

\section{6. - DISTRICT DU DIBEN (VI, 40 à 43).}

De Ty Lousou au port de Primel. Falaises rocheuses, essentiellement constituées par le granite fin de Primel qui enclave quelques vastes panneaux de granite porphyroïde de Saint-Samson. Plages peu nombreuses, généralement exposées au nord-ouest, souvent ourlées par un cordon de galets bien roulés (Le Guerzit), ou, exceptionnellement par une dune (Saint-Samson). Ce district se caractérise par ses faibles teneurs en minéraux lourds (parmi les plus faibles de la baie de Morlaix, avec celles du district de Roscoff) et la rareté des concentrations (la plage de SaintSamson mise à part). On constate une nette augmentation du pourcentage en tourmaline, des teneurs assez fortes en zircon, un prélèvement avec une teneur en monazite exceptionnellement élevée (près de $15 \%$ !), mais inexpliquée à ce jour, un échantillon relativement riche en staurotide $(4,3 \%)$, de fortes teneurs en ilménite (jusqu'à $85,3 \%$, teneur moyenne $=62,1 \%$ ) et, par rapport au district précédent, une chute très nette des pourcentages en amphibole $(8,5 \%)$ et en épidote $(4,6 \%)$. La présence de minéraux très altérables, comme le mispickel et la chalcopyrite, est un fort argument pour l'origine locale d'une partie des dépôts sableux. Si, dans l'ensemble, les individus anguleux dominent (même sur une grève aussi exposée que celle du Guerzit), les minéraux plus ou moins usés sont localement abondants (Saint-Samson). La réapparition de la tourmaline (qui n'est que partiellement d'origine locale) et surtout de la staurotide (inconnuc en place) démontre ici un apport en provenance de l'Ouest. 


\section{7. - DISTRICT DE PRIMEL (VII, 44 à 47).}

Ce petit district qui correspond à l'anse de Primel, est bordé par le gabbro (épidioritisé) de Saint-Jean-du-Doigt, recoupé, au nord, par le granite à grain fin de Primel. Grèves rares, de petite dimension, souvent encombrées de blocs. On retrouve ici des pourcentages en M.L. relativement élevés $(45,9 \%)$. Le spectre minéralogique est dominé par l'ilménite (jusqu'à près de $93 \%$ !, soit la plus forte teneur observée en baie de Morlaix) et accessoirement par l'amphibole et l'épidote, à teneur variable, mais toujours significative. Très caractéristiques sont la rareté de la tourmaline et du grenat, et l'absence de la staurotide. Le cortège minéralogique et la morphologie anguleuse de l'ilménite (minéral dominant) attestent l'origine locale des sables lourds, en provenance manifeste des gabbros.

\section{II. - MODAlites DE LA CONCENTRATION.}

L'analyse qui précède a souligné, à plusieurs reprises, l'origine le plus souvent proximale des sables lourds concentrés localement sur les plages de la baie de Morlaix. En première approximation, et en se limitant aux minéraux principaux, chaque district mis en évidence offre une individualité bien marquée qui reflète une zone-source prédominante. Les placers appartiennent souvent à la " lignée pure ", mais les " lignées croisées ", avec processus d'hybridation, sont également observées. Les exceptions à l'origine proximale sont relativement rares et facilement interprétables. Les apports accidentaux (district I et pro parte district VI) sont marqués en particulier par la tourmaline et, éventuellement, la staurotide. Certains minéraux, tels le disthène et la sillimanite (liés, comme la staurotide, aux formations métamorphiques du Léon), fréquemment décelés mais toujours en faible quantité, traduisent aussi, à l'évidence, la présence d'un apport occidental dans les secteurs orientaux (Petit-Trégor). L'absence presque totale de sillimanite dans le district III marque les limites de cet apport dans les zones les plus profondes de la baie. L'andalousite fournit un exemple cle halo de dispersion autour de la roche-mère. Si ce minéral est omniprésent en baie de Morlaix (40/47 prélèvements), les teneurs significatives ( 6 échantillons : 0,8 à $7,1 \%$ ) reproduisent approximativement, en l'élargissant, la zone d'affleurement des schistes métamorphisés par le granite. Un exemple de double source proximale est donné par l'occurrence de Créac'h André (II,4) : amphibole (dominante) en provenance de la diorite ; staurotide et grenat (accessoires) origınaires des micaschistes. $\mathrm{Au}$ total, les sept districts mis en évidence correspondent à autant de petites cellules dont les communications avec les cellules voisines restent, dans l'ensemble, peu importantes. Elles ne sont toutefois pas inexistantes comme l'ont montré transitions, halos de dispersion et apports occidentaux.

A l'échelle de l'ensemble de la baie, certains minéraux lourds permettent d'effectuer des regroupements plus généraux. La scheelite est essentiellement déceléc dans les quatre districts occidentaux (16 sur 17 échantillons minéralisés). Le grenat, omniprésent, offre une répartition très anomalique, avec des districts à teneur élevée (I et II) et des districts à teneur très faible (VII) ou faible (V, VI, IV), mis à part quelques points exception- 
TABLE:All II - Teneurs en $\mathrm{CO}_{3} \mathrm{Ca}$ de sables des estrans de la baie de Morlaix.

\begin{tabular}{|c|c|c|c|c|}
\hline & LOCALISATION & DISTRICT & $\begin{array}{l}\text { IENEUR ET NOMBRE } \\
\text { D'ECHANTILLONS }\end{array}$ & REFERENCES \\
\hline 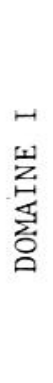 & $\begin{array}{l}\text { Penprat } \\
\text { Graou } \\
\text { Penquer } \\
\text { Ile Callot } \\
\text { Barnenez } \\
\text { Kernelehen } \\
\text { Penallan }\end{array}$ & $\begin{array}{l}\text { II } \\
\text { II } \\
\text { III } \\
\text { IV } \\
V \text { (Sud) } \\
V \text { (Sud) } \\
V \text { (Sud) }\end{array}$ & $\begin{array}{ll}3,0 \% & (36) \\
3,6 \% & (9) \\
1,8 \% & (4) \\
2,3 \% & (83) \\
2,0 \% & (1) \\
2,5 \% & (3) \\
1,3 \% & (5)\end{array}$ & $\begin{array}{l}\text { M.M. Chauris (inédit) } \\
\text { M. Masson (1969) } \\
\text { M.M. Chauris (inédit) } \\
\text { R. Loarer (1975) } \\
\text { A.M. Cadiou (1969) } \\
\text { A.M. Cadiou (1969) } \\
\text { A.M. Cadiou (1969) }\end{array}$ \\
\hline 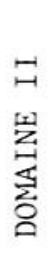 & $\begin{array}{l}\text { Sterec (Sud) } \\
\text { Sterec (Nord) } \\
\text { Ty Lousou } \\
\text { Saint-Samson } \\
\text { Le Guerzit }\end{array}$ & $\begin{array}{l}\text { V (Nord) } \\
V \text { (Nord) } \\
V \text { (Nord) } \\
\text { VI } \\
\text { VI }\end{array}$ & $\begin{aligned} 19,1 & \% \\
23,0 & \%(14) \\
7,5 \% & (1) \\
4,1 \% & (2) \\
21,6 \% & (1)\end{aligned}$ & $\begin{array}{l}\text { A.M. Cadiou (1969) } \\
\text { A.M. Cadiou (1969) } \\
\text { M.M. Chauris (inédit) } \\
\text { M.M. Chauris (inédit) } \\
\text { A.M. Cadiou (1969) }\end{array}$ \\
\hline
\end{tabular}

nels facilement expliqués (échantillon 30 par exemple). A priori, deux origines sont possibles pour les grenats : les micaschistes (ex : Grève du Man); les formations aplito-pegmatitiques associées avec leucogranites (environ de Roscoff, Chauris, en préparation). La staurotide présente une répartition analogue à celle du grenat (concentration dans les districts I et II). I'association grenat-staurotide montre que dans le district II tout au moins, le grenat provient essentiellement des micaschistes. Grenat (quelle qu'en soit l'origine) et staurotide caractérisent à l'évidence le Pays de Léon. Inversement, l'épidote est toujours présente dans les secteurs orientaux (Petit-Trégor), parfois avec des teneurs élevées (district V) alors qu'elle est le plus souvent sporadique dans les secteurs occidentaux (Pavs de Léon). Par ailleurs, le spectre minéralogique est plus riche dans le domaine ouest (Pays de Léon, districts I à IV) que dans le domaine est (Petit-Trégor, V à VII) (en moyenne, respectivement 17 et 14 espèces). Cette différence correspond, en partie, à la monotonie plus grande des roches-mères du Petit-Trégor. Au total, l'opposition entre Léon et Trégor, sensible dans la répartion de nombreux minéraux lourds des plages, reflète la différence de composition pétrographique entre ces deux domaines géotectoniques.

Certaines roches, de par leur nature pétrographique, sont particulièrement favorables à la libération d'une grande quantité de M.L. En baie de Morlaix, on citera plus particulièrement les amphibolites gneissiques (ilménite, district III), les gabbros et épidiorites (ilménite, district VII), diverses roches vertes (amphibole, épidote, district V), les diorites (amphibole, sphène, district II), les micaschistes (grenat et staurotide, district II), etc... L'influence prépondérante de certaines roches-mères (amphibolites, gabbros...) induit le cachet dominant des sables lourds. Ainsi le minéral lourd de loin le plus répandu est l'ilménite et c'est lui qui confère aux 
sables des placers leur couleur noire. Sur les 47 échantillons analysés, 45 ont montré des teneurs significatives, allant de 3,5 à 92,8\% (moyenne = $54,2 \%$ ). Ces résultats contrastent vivement avec ceux obtenus immédiatement plus à l'ouest (environs de Roscoff) où la teneur moyenne en ilménite de 8 échantillons n'est que de $11,5 \%$, soit près de cinq fois moins (Chauris, en préparation). Inversement, l'absence ou la rareté de certains minéraux dans quelques districts traduit directement l'absence ou la rareté de roches-mères favorables : c'est, en particulier, le cas pour la tourmaline (district III) (amphibolites gneissiques) et VII (gabbro) ; le grenat (district V et surtout VII [roches-vertes]) ; la staurotide (districts V, VI et VII); l'épidote (district IV) ; la monazite (districts V, VII), etc...

L'acheminement des minéraux lourds, dispersés dans les roches-mères, jusqu'à leur dépôt dans les placers littoraux, s'effectue selon des modalités complexes (apport sur les estrans, concentration sur les hauts de plages).

Apport des ruisseaux côtiers - Outre les deux grandes rias de la Penzé et de la Rivière de Morlaix, de multiples ruisseaux se jettent en baie de Morlaix. Les prospections alluvionnaires du B.R.G.M. (Guigues et Devismes, 1969) ont montré (ici comme partout ailleurs dans le Massif armoricain), la présence, dans les sables fluviatiles, de nombreux minéraux lourds en cours de transit. Le cortège minéralogique de ces sables reproduit étroitement - dans la grande généralité des cas - les associations des bassins versants (origine proximale). Actuellement, les apports de ces ruisseaux à la mer semblent faibles, mais ils ont été certainement élevés lors des débâcles périglaciaires würmiennes. La petite rivière qui se jette dans l'anse du Diben draine, en amont, une importante zone stannifère qui a fait, voici une douzaine d'années, l'objet d'une exploitation alluvionnaire. Les tencurs en cassitérite décroissent vers l'aval. Dans la partie méridionale de l'anse du Diben (prélèvement 45 et 44 ), ce minéral n'a été décelé qu'en microtraces ; il n'a pas été observé dans la partie septentrionale de l'anse (prélèvements 46 et 47 ).

Apporc des formations meubles en voie d'attaque par la mer. - C'est ici que paraissent se situer les principales sources de M.L. actuellement concentrés sur les estrans de la baie de Morlaix.

(a) La destruction des plages anciennes. D'innombrables restes de plages "bas-normaniennes" (Eemien) sont situés un peu au-dessus des hautes mers actuelles, sous les coulées de solifluxion würmiennes (Wechselien) qui les remanient. Les occurrences sont surtout formées de galets. Un des objectifs des futures recherches sera de caractériser le spectre des M.L. des anciens dépôts sableux de la baie. Il paraît évident que les résultats obtenus plus à l'ouest dans le Léon se retrouveront ici. Au Vougo (à l'ouest de Guissény), B. Hallégouc̈t (1971) a montré le parallélisme entre les associations minéralogiques de la plage ancienne et celles de la plage actuelle. Le même parallélisme a été également noté à Brignogan (Duplaix et Boillot, 1968).

(b) Le lessivage des altérites. Les travaux de Berthois (1935) ont souligné l'importance de l'arénisation dans la libération des minéraux lourds. Les exemples sont innombrables en baie de Morlaix (amphibolite gneissique à ilménite du district III, diorite de Créac'h André, district II, etc...). 
(c) Déblayage du head et des limons würmiens. L'origine essentiellement locale des limons du Nord-Finistère, a été établie par l'étude des M.L. (Berthois et Milon, 1932). Par rapport aux arènes, les formations solifluées sont nettement enrichies en M.L. (Berthois et Dangeard, 1929). En baie de Saint-Brieuc, le pourcentage en minéraux lourds des limons va de $0,60 \%$ à plus de $2 \%$ (Berthois, 1937). Au pied des falaises limoneuses de Keryenevet (district III), en voie d'éboulement, il a été possible d'observer (hiver 1981), des concentrations élevées en minéraux lourds en cours de lessivage. Au total, l'ampleur des coulées de solifluxion et la rapidité de leur attaque par la mer font de ces formations périglaciaires, l'une des sources majeures en minéraux lourds.

(d) Recul des dunes. La destruction aisée de ces dépôts meubles contribue également à alimenter les plages. Ce processus est relativement secondaire, mais non négligeable, en baie de Morlaix où les dunes sont peu nombreuses, et de faible puissance, mais semble par contre très actif plus à l'ouest dans les environs de Roscoff-Santec (Francis-Bœuf, Bourcart, Duplaix, 1949), de Brignogan et de Landunvez (Duplaix et Boillot, 1968) où le spectre des M.L. de l'estran reflète nettement celui des hautes plages dunaires ou des dunes anciennes.

L'énorme stock de M.L. ainsi libéré est étalé sur les estrans (avec les minéraux légers auxquels les M.L. sont initialement associés), puis, en partie, concentré localement sur le haut des plages, dans un environnemont de haute énergie nécessaire à la séparation. Dans les zones de moindre énergie, les M.L. restent dilués dans la masse de minéraux légers. Ce fait a été observé en plusieurs points, par exemple sur les parties inférieures du vaste estran sableux de la Grève Blanche en Carantec (district IV). Les minéraux disséminés forment alors des " placers potentiels " (Cronar, 1980). L'effet saisonnier est ici essentiel : en hiver, (période des plus fortes tempêtes), on a noté une nette augmentation des concentrations en M.L. sur les parties hautes des estrans, du fait de l'éloignement plus énergique des minéraux légers. Ainsi est soulignée la nécessité d'observations répétées, à diverses saisons et après des périodes de temps différents.

Les processus de concentration dans les placers littoraux - véritables pièges à minéraux - s'avèrent très complexes. A la dérive littorale, qui peut induire déjà un premier tri sélectif, se superpose l'énergie des vagues dans la zone de déferlement induisant des mouvements de type "batée " et " jig * (éloignement des minéraux légers et chute plus rapide des M.L.) (Burns, 1979). L'ampleur du fetch constitue ainsi un facteur de premier ordre. Ces processus hydrodynamiques agissent sur les propriétés spécifiques de flottabilité des différents minéraux, liées à leur densité, mais également à leur forme (opposition entre les grenats plus ou moins globuleux et les amphiboles en tablettes grossières, etc...). L'influence de la morphologie littorale sur le dépôt des M.L. est soulignée par de multiples exemples : le plus souvent, seule l'une des parties de la plage est minéralisée (ainsi à Penn ar Warem, district IV, la partie septentrionale, etc...). Dans quelques cas (prélèvement 20), les concentrations de M.L. sont étalées directement sur le bed-rock. Plus souvent, les couches minéralisées alternent avec les couches stériles. Le problème de la stabilité du cortège des plages n'a pu encore être abordé : il demandera de nombreuses analyses échelonnées sur plusieurs années. 
Au total, les concentrations en M.L. de la baie de Morlaix peuvent présenter diverses filiations, mais où domine une origine proximale impliquant une migration limitée (districts I et VI mis à part). L'indice de jeunesse de ces sédiments est attesté par le fort pourcentage de minéraux très arıuleux et par la présence, en quantité notable, d'une espèce relativement altérable comme l'amphibole (hornblende). Ainsi est souligné le caractère immature des placers.

Peut-on extrapoler les résultats fournis par l'étude des minéraux lourds à la masse du sable des plages constituée essentiellement de minéraux légers (quartz. surtout) ? A la différence de densité s'ajoute la faiblesse du rapport minéraux lourds/minéraux légers (de l'ordre de 1/50 à 1/100 en général). A l'évidence, les possibilités de migration des minéraux légers sont certainement beaucoup plus importantes que celles des minéraux lourds. Ce problème méthodologique, qui n'a pu être entièrement résolu dans le cadre de la baie de Morlaix, peut être toutefois abordé de manière indirecte.

La prédominance de l'influence des sources on shore sur l'origine du sable des plages, fortement suggérée par l'étude des concentrations de M.L. dans les micro-placers, est également appuyée par l'examen des minéraux constitutifs essentiels des sables prélevés en dehors des zones de concentrations quelque peu anormales que constituent les placers. Les analyses révèlent, en bien des cas, un reflet direct du substratum voisin, surtout en ce qui concerne les parties grossières $(>0,315)$. Ainsi, à La Graou (district II), les principaux minéraux du granite à deux micas de Saint-Anne se retrouvent dans les sables : quartz $(76 \%)$, feldspath $(9 \%)$, muscovite $(7 \%)$, biotite $(5 \%)$, (Masson, 1969). A Callot (district IV), où affleure largement le granite à biotite, les pourcentages sont les suivants : quartz $(84 \%)$, feldspath $(6,5 \%)$, biotite $(4,5 \%)$, (Loarer, 1975). De telles compositions, avec feldspath et biotite en proportion élevée, révèlent des sables immatures d'origine proximalc. Il en cst de même sur les bords de la Rivière de Morlaix (tant au nord de Locquénolé qu'au nord de Dourduff), où les sables sont très riches en minuscules débris schisteux, provenant, à l'évidence, de l'encaissement immédiat, etc... Ces conclusions sur l'origine on shore proximale sont également confirmées par les observations morphoscopiques (mesures effectuées sur les quartz, $>0,315$ ) qui indiquent une très grande proportion de N.U. : à Callot, par exemple. district IV, de l'ordre de $90 \%$ et souvent près de $100 \%$ (Loarer, 1975), confirmant la jeunesse du sédiment et une provenance locale.

Les données de la calcimétrie appuient aussi largement l'origine essentiellement on shore proximale de nombreuses accumulations sableuses des estrans de la baie. Dans les régions totalement dépourvues d'afleurement calcaire, comme dans le territoire étudié ici, la teneur en $\mathrm{CO}_{3} \mathrm{Ca}$ traduit uniquement un apport organogène. Les résultats obtenus par plusieurs études détaillées (Masson, 1969 ; Cadiou, 1969 ; Loarer, 1975 ; MM. Chauris, inédit) s'avèrent très instructifs. Le tableau II met en évidence deux domaines différents : un domaine sud-ouest (districts II, III, IV et V [partie sud]), très étendu, caractérisé par des teneurs très faibles en $\mathrm{CO}_{3} \mathrm{Ca}$ (tencurs moyennes comprises entre 1,3\% et 3,6\%); un domaine restreint, essentiellement limité à la partie nord-est de la baie (district V [partie nord] et district VI où les teneurs peuvent dépasser $20 \%$, soit, approxi- 
mativement dix fois plus élevées que dans le premier domaine. (Le district I n'a pas été analysé en détail au point de vue calcimétrie ; des observations préliminaires, indiquent de basses teneurs. La faible teneur des échantillons de Saint-Samson est due au fait qu'une part importante du sable de la plage provient de la dune bordière). Dans le premier domaine, l'apport marin coquillier sur les hauts de plages apparaît négligeable alors qu'il devient important dans le second domaine. Dans le détail, on observe. en règle générale, une augmentation des teneurs en descendant sur l'estran : ainsi, à Penprat (MM. Chauris, inédit), la succession suivante a été notée de haut en bas (équidistance des prélèvements : 40 m) : $1,5 \%-5,1 \%-7,1 \%-9,2 \%$. Les données obtenues sont en bon accord avec celles présentées par Francis-Bœuf et al (1949) pour l'anse de l'Aber en Roscoff (teneur moyenne $<2 \%$ sur les parties hautes des plages, avec augmentation progressive sur les parties basses de l'estran $(>6 \%)$.

L'examen de la carte sédimentologique de la baie de Morlaix dressée par Boillot $(1961,1964)$ va également dans le même sens. Une partie importante de la baie est occupée par des sédiments littoraux terrigènes (ou, localement, par du maërl dont l'origine in situ est bien établie). Les sables zoogènes ne deviennent prépondérants que dans la partie nord-est de la baje, audelà d'une ligne Le Menk - Ile Verte - Les Roches Jaunes. Encore doit-on remarquer que toute la partie sud-ouest de ces sables est constituée par des débris de coquilles d'organismes vivants sur le littoral ou près des côtes (moules, etc...)., et a donc, en ce sens, la même signification, au point de vue origine, que les sables détritiques siliceux (origine proximale). Ce n'est qu'à l'extrémité nord-est de la baie (dune hydraulique du Rater, environs de la Pierre Noire) que les sables zoogènes d'origine plus lointaine, prennent de l'importance. Par ailleurs, la présence de maërl vivant dans les zones de Guerhéon, Ricard et du Taureau pourrait également confirmer l'absence d'apport sableux significatif dans les zones de la baie oì se développe cette algue.

Des conclusions semblables (origine on shore proximale des sédiments de la plus grande partic des zones intertidales de la baie de Morlaix) paraissent pouvoir également s'appliquer aux vases qui affleurent largement, à basse mer, sur les rives des deux estuaires et leurs diverticules. Dans son étude sur les vases de La Penzé, Auffret (1968) a conclu que le quartz (minéral constitutif essentiel de la vase) provient du remaniement des coulées de solifluxion wüirmiennes. Une telle origine proximale est également appuyée par l'étude des M.L. (avec grande abondance de l'amphibole, minéral essentiel des amphibolites de l'embouchure de la ria). C'est à la même conclusion qu'étaient arrivés Guilcher et Berthois (1957), Guilcher (1967) pour d'autres estuaires finistériens (Guilcher et al., 1982).

\section{CONCLUSION}

L'étude des micro-placers littoraux de la baie de Morlaix doit être considérée comme une contribution - grâce à l'étude des minéraux lourds à la dynamique si complexe des dépôts sableux sur la partie haute des estrans, dans une profonde échancrure littorale, soumise à un régime de fortes marées (macrotidal bay). En baie de Morlaix. les placers littoraux 
peuvent être regroupés en un ensemble de sept districts (petites cellules) présentant des associations minéralogiques différentes. L'essentiel des sources est d'origine proximale ; le transport reste faible. A l'évidence, la sédimentation s'est effectuée ici principalement en dehors des grands courants de circulation de la Manche (transit général d'ouest en est, au large de Roscoff, Le Gorgeu et Boillot, 1964). Les «entrées » en provenance de l'ouest sont essentiellement limitées aux districts I et VI. Du fait de la dérive littorale nord-sud, dans la baie (courants de flot supérieurs aux courants de jusant), les " sorties " sont probablement restreintes. Ces polarités ouest-est et nord-sud sont liées à l'hydrodynamisme des marées. Les données tirées de l'étude des minéraux lourds, complétées par les résultats de la calcimétrie, s'accordent pour classer la partie supérieure des estrans de la baie de Morlaix en deux domaines différents : un domaine très étendu au sud-ouest d'une ligne Roscoff - Sterec, caractérisé essenticllement par des apports on shore locaux (fraction lithoclastique très dominante) ; un domaine plus réduit, au nord-est où, à l'apport on shore indiscutable (établi par l'étude des minéraux lourds) s'ajoute un apport off shore (démontré par l'examen des minéraux lourds et par la calcimétrie [présence d'une fraction bioclastique non négligeable]). La différence entre les deux domaines ne paraît pas due au fetch, mais principalement à l'influence du courant de flot et ainsi liée à la dérive résiduelle orientée d'ouest en est au large de Roscoff. Au total, dans le problème de la balance sédimentologique des sources respectives "off shore" - " on shore ", les résultats obtenus privilégient l'influence prédominante des sources " on shore".

Les associations mineralogiques et la morphoscopie soulignent le caractère typiqucment immature des placers dont l'origine est, le plus souvent, autochtone à subautochtone. Les minéraux lourds, dérivés essentiellement des arènes, du head et des limons périglaciaires portent aussi la marque d'une origine continentale. La mer actuelle ne fait que déblayer le manteau d'altérites et de coulées de solifluxion. Sous cet aspect, l'origine première de minéraux lourds concentrés dans les placers littoraux de la baie de Morlaix n'es! pas fondamentalement différente de celle des placers fluviatiles de la région voisine de Saint-Renan dans le Bas-Léon (Guilcher et al., 1961 ; Moussu, 1963) où les eaux fluviales n'ont fait que trier et enrichir en M.L. les arènes et les coulées de solifluxion étalées dans la large vallée de l'Aber I1dut. Dans les deux cas, le climat périglaciaire würmien est responsable de l'entraînement vers les points bas, d'énormes volumes de formations meubles contenant des M.L. dispersés. Comme le pouvoir concentrateur de la mer par les vagues, sur les parties hautes des estrans, est beáucoup plus efficace que celui d'une rivière comme l'Aber Ildut, mème en crue, les concentrations locales sont toujours beaucoup plus élevées sur le haut des estrans que dans les sables fluviatiles. Toutefois, la teneur moyenne n'est ici sans doute pas plus forte.

Des modifications anthropiques (murs, enrochements...) sont entreprises actuellement un peu partout en baie de Morlaix, en vue de tenter d'enrayer les processus d'érosion localement spectaculaires dans les arènes, le head, les limons et les dunes. En tarissant ainsi peu à peu les sources des sables d'origine terrigène, ces travaux risquent progressivement d'appauvrir les plages. Cette déduction illustre, une fois de plus, 
l'équilibre délicat entre les travaux de l'Homme et les forces de la $\mathrm{Na}$ ture. Une telle conclusion ne s'applique toutefois que partiellement aux zones grandes ouvertes vers le large où, à l'apport de sables terrigènes s'ajoute un apport organique important (district VI en particulier).

Les conclusions formulées dans ce travail sur la baie de Morlaix seront élargies ultérieurement par les résultats de l'étude (toujours axée sur les cortèges de minéraux lourds des placers littoraux) des deux régions voisines : d'une part, à l'est, la baie de Lannion, beaucoup plus exposée aux influences du large que la baie de Morlaix ; d'autre part, à l'ouest, la presqu'île de Roscoff - Santec et l'île de Batz, situées en avant de la ligne générale du rivage. La confrontation des résultats obtenus par l'examen de ces trois régions voisines, mais différentes, permettra de proposer un certain nombre de règles de portée plus générale.

Au terme de telles investigations, peut-être n'est-il pas téméraire d'espérer la possibilité de quantifications des transports, ce qui serait tout à fait dans la ligne des préoccupations actuelles des géomorphologues.

\section{Remerciements}

Nous remercions vivement $H$. Chevance, chef du laboratoire de minéralogie du B.R.G.M. à Nantes, qui a effectué les analyses de minéraux lourds ; A. Guilcher, qui nous a amené à préciser l'intérêt géographique de notre texte ; J.L. Travers, qui a redessiné la carte ; et D. Rachebœuf, qui a dactylographié le manuscrit. Ce travail a été entrepris dans le cadre du G.I.S. 410012 "Océanologie et Géodynamique ».

\section{BIBLIOGRAPHIE}

Auffret (G.) (1968). Contribution à l'étude sédimentologique de la ria de La Penzé (Finistère). Thèse $3^{e}$ cycle, Paris, $127 \mathrm{p}$.

AtrFRet (G.) et Douville (J.L.) (1974). Observations sur la dynamique des sables de la Pierre-Noire (Baie de Morlaix). Bull. Rech. Géol. Min. 2 (IV), p. 5-18.

BAAK (J.A.) (1936). Regional petrology of the Southern North Sea. These, Wageningen, $127 \mathrm{p}$.

BARROIS (Ch.) (1909). Carte géologique au 1/80 000 Lannion.

Brauchamp (P.) de (1914). Les grèves de Roscoff. Le Chevalier édit., Paris.

BERThois (L.) (1935). Recherches sur les minéraux lourds des granites de la partie orientale du Massif armoricain. Mém. Soc. Géol. Minéral. Bretagne, 2, $190 \mathrm{p}$.

Berthois (L.) (1937). Contribution à l'étude des limons de Bretagne. Bull. Soc. scientif. Bretagne, XIV, p. 53-62.

Berthois (L.) et DANGEARd (L.) (1929). Formations quaternaires aux environs du Conquet et de Lanildut (Finistère). Bull. Soc. Géol. Minéral. Bretagne X, p. 44-77.

Berruois (L.) et Furnestin (J.) (1937). Etude des sédiments dragués par le « Président Théodore Tissier " (Plateau continental celte, Manche et Mer du Nord). C.R. Acad. Sci., 240, p. $1876-1878$.

Berithois (L.) et Milon (Y.) (1932). Note préliminaire sur les limons quaternaires du Finistère. C.R. somm. Soc. Géol. Fr., p. 53-55.

BrLly (C.) (1964): Etude minéralogique des sédiments côtiers de la Manche entre l'estuaire de la Dives et l'estuaire de la Somme. Rev. Géogr. phys. et Géol. dynam. (2), VI, (2), p. 123-154.

Boll. or (G.) (1961). La répartition des sédiments en baie de Morlaix et en baie de Siec. Cohiers de Biologie Marine, II, p. 53-66.

Borllor (G.) (1964). Géologie de La Manche occidentale. Ann. Institut océanogr. XLII, 220 p. 
Bolreart (I.) (1948). Le Quaternaire des Grèves de Roscoff (Finistère). Bull Soc. Géol. Fr., p. 181-197.

Bourcart (J.) (1949). Géographie du fond des mers. Payot, Paris, 307 p.

Burns (V.M.) (1979). Marine placer minerals. In vol. 6 « Marine Minerals », Reviews in Mineralog. - Mineral. Soc. America, p. 347-380.

Cabioch (L.) et Douville (J.L.) (1979). La circulation des eaux dans la baie de Morlaix et sris abords : premières données obtenues par suivis de flotteurs dérivants. Trav. Stat. Biol. Roscoff, N.S., XXV, p. 11-20.

CAlsou (A.M.) (1969). Les accumulations de la baie de Morlaix entre Kerarmel et la pointe d'Annalouesten. Mém. Maîtrise Géogr. Univ. Brest, inédit, 41 p.

CHASsÉ (Cl.) (1972). Economie sédimentaire et biologique (production) des estrans méubles des côtes de Bretagne. Thèse d'Etat, Paris, 293 p.

Chauris (L.) (1972). La limite Léon-Trégor et l'origine de la baie de Morlaix (Massif armoricain). C.R. somm. Soc. Géol. Fr., p. 137-139.

Chauris (L.) (1975). Minéralisations stanno-wolframifères dans le granite de Carantec (litetagne). CR. Acad. Sc., Paris, 280, p. 2421-2424.

Chal'RIS (L.) (1978). Succession des intrusions dans le complexe granitique tardi-hercynien de la baie de Morlaix (Massif armoricain). C.R. Acad. Sc., Paris, 286, p. 253-256.

Chalris (L.) (1982a). Les sables lourds des plages du Mor Bras. Introduction à l'étude des placers littoraux en Bretagne méridionale. Bull. Soc. Sc. nat. Ouest de la France, $n^{\text {lle }}$ série, $I V, 1$, p. 1-58.

Chлukis (L.) (1982b). A la recherche des placers littoraux : concentration de sables lourds sur les plages du Nord-Finistère (Massif armoricain). $107^{\circ}$ Congrès nat. Soc. sav., Brest, Sciences, fasc. III, p. 119-130.

Chauris (L.) (1982c). Placers littoraux à ilménite et magnétite : les sables noirs des plages de Saint-Quay-Portrieux (Massif armoricain). Mém. Géol. Univ. Dijon, 7, p. 301-311.

Chaukis-Norroy (M.M.) (1973). Formations littorales de Saint-Pol de Léon. Penn ar Bed, 75 , p. $130-141$.

Cronan (D.S.) (1980). Underwater minerals. Academic Press, London, 362 p.

DeunfF (J.) (195.1). Observations générales sur la pointe de Primel. 76" Congrès Soc. sav., p. $125-129$.

Divismes (P.) (1978). Atlas photographique des minéraux d'alluvions. Mém. B.R.G.M., $n^{\nu} 95,20 \overline{\mathrm{p}} \mathrm{p}$.

Duplaix (S.) (1946). Les minéraux lourds de quelques sables du Finistère. C.R. somm. Soc. Géol. Fr., p. 131-133.

Diplaix (S.) et Boillot (G.) (1968). Sur la minéralogie et l'origine des sables siliceux contenus dans les sédiments de la Manche occidentale. Rev. Géogr. phys. et Géol. dynam. (2), $X, 2$, p. 147-161.

Francis-Bauf (Cl.) (1947). Recherches sur le milieu fluvio-marin et les dépôts d'estuaire. Ann. Inst. Océanogr., XXIII, 3, p. 149-344.

Francis-Beeuf (Cl.), Bourcart (J.) et Duplaix (S.) (1949). La couverture sédimentaire de l'Aber de Roscoff (Finistère). Sédiment et Quaternaire. I.ed-Sam Edit. Bordeaux, p. 21-48.

Girfsse (P.) et LARSONneur (Cl.) (1970). La succession des apports sableux en Manche centrale grâce à l'étude des minéraux lourds. Rev. Géogr. phys. et Géol. dynam., (2), $X I I, 1$, p. 41-62.

Guigues (J.) et Devismes (P.) (1969). La prospection minière à la batée dans le Massif armoricain. Mćm. B.R.G.M., $n^{\circ} 71,172$ p.

Guij.cher (A.) (1954). Morphologie littorale et sous-marine. Presses Univers. Fr., Paris. 214 p.

GuIlcher (A.) (1967). Origin of sediments in estuaries. In « Estuaries », Am. Ass. Adv. Sci., p. 149-157.

Guilcher (A.), Andrade (B.) et Dantec (M.H.) (1982). Diversité morpho-sédimentologique des estuaires du Finistère. Norois, 114, p. 205-228.

Guilcher (A.) et Berthois (L.) (1957). Cinq années d'observations sédimentologiques dans quatre estuaires-témoins de l'Ouest de la Bretagne. Rev. Géomorph. Dyn., 8, p. 66-86.

Guilcher (A.), Ters (M.) et BonNet (J.Y.) (1961). Données récentes sur l'évolution morphologique des vallées bretonnes : Oust et Vallée de Saint-Renan. Comité tr. hist. et scient. Bull. Sect. Géogr. Impr. Nation., Paris, 74, $53 \mathrm{p}$.

Hallégouet (B.) (1971). Le Bas-Léon (Finistère, France). Etude géomorphologique. Thèse $3^{e}$ cycle, Brest, $260 \mathrm{p}$. 
L.tFoN (M.) (1953). Recherches sur les sables côtiers de la Basse-Normandie et sur quelques conditions de leur peuplement zoologique. Ann. Inst. Océanogr., 28, 3, p. 113-161.

L.ARsonneur (C.), VASLet (D.) et AufFret (J.P.) (1978). La carte des sédiments superficiels de la Manche au 1/500 000. Bur. Rech. Géol. Min. édit., Orléans.

LE GoRgeu (J.P.) et Boillot (G.) (1964). La migration des sables marqués au large de Roscoff. C.R. Acad. Sc., Paris, 259, p. 4082-4085.

LoARI:R (R.) (1975). Ile Callot. Morphologie et sédimentologie. Mém. de Maîtrise Géogr. Univ. de Brest, 126 p. +73 p. (annexes).

Masson (M.) (1969). La Graou sur la côte de Saint-Pol de Léon. Sédimentologie et cartographie. Mém. de Maîtrise Géogr., Univ. de Brest, 62 p., inédit.

MOORE (J.R.) (1978). Marine placers : exploration problems and sites for new discoveries. In « Ressources minérales sous-marines ". C.R. Séminaire International Germinal, Orleans, p. 131-163.

Moussu (R.) (1963). La campagne de recherches $1958-1960$ dans la région stannifère de Suint-Renan. Rapport CoMIREN, inédit.

Nurkoy (M.M.) (1969). La Graou, accumulation de galets en la commune de Saint-Pol de Léon. Mén. Maîtrise Géogr., Univ. de Brest, 92 p., inédit.

SANiJReA (A.) (1958). Contribution à la lithologie de la côte nord de la Bretagne : de l'île de Sieck à la baie de Perros. Bull. Serv. Carte Géol. Fr., LVI, 258, p. 505-616.

SeRvice hydRographiQue de la MARINe (1953). Courants de marée dans la Manche et sur les côtes françaises de l'Atlantique, no 550, Paris, 276 p. Carte marine : De la pointe de Bloscon à la pointe de Primel - Baie de Morlaix. $n^{\circ} 5827$ à $1 / 20000^{\circ}$.

Tixziront (M.) (1978). La démarche de la France dans la recherche des placers sur les plateaux continentaux. In * Ressources minérales sous-marines ». C.R. Séminaire International Germinal, Orléans, p. 167-184.

\section{RESUME}

L'étude des cortèges de minéraux lourds (47 prélèvements) concentrés sur les plages de la baie de Morlaix, profonde échancrure littorale de la Manche occidentale, permet de mettre en évidence sept districts différents. Dans l'ensemble, les placers littoraux présentent une origine proximale. Les sables lourds proviennent essentiellement de l'érosion et du tri par la mer, de dépôts continentaux récents (arènes, formations périglaciaires ou heud). Les apports extérieurs à la baie restent limités, sauf à son extrémité nord-ouest et surtout dans sa partie nord-est. L'origine proximale d'une grande partie des sables contrôle le devenir des plages actuellement soumises à des modifications anthropiques.

\section{SUMMARY}

Investigations carried out upon heavy minerals (47 samples) concentrated along the beaches of the bay of Morlaix, a large coastal indentation in the Western Channel, have displayed seven different districts. As a general rule, the coastal placers present a proximal origin. The heavy sands come essentially, by marine erosion and sorting, from late continental sediments (arena and periglacial deposits, head). The outer contributions are small, except for the northwestern end of the bay and especially its northeastern part. The proximal origin of an important part of the sands could account for the future of the beaches, at present time altered by anthropic modifications. 